\title{
Timing Acquisition for Multi-User IDMA
}

\author{
Bathiya Senanayake \\ Research School of Information Sciences and Engineering \\ Australian National University, \\ ACT, Australia 0200 \\ Email: bathiya.senanayake@mail.rsise.anu.edu.au
}

\author{
Mark, C. Reed and Zhenning Shi \\ National ICT Australia \\ Australian National University, \\ ACT, Australia, 2601 \\ Email: \{mark.reed, zhenning.shi\}@nicta.com.au
}

\begin{abstract}
Acquisition of the timing in an IDMA system must take place before signal detection and decoding are performed. Acquisition in the presence of severe multiple-access interference with time varying codes makes the task even more difficult. Inefficient designs cause a large number of false alarms and/or missed detections. This paper applies a powerful acquisition technique to IDMA systems in the uplink. Under high multiple access interference conditions conventional acquisition techniques used for DS-CDMA systems simply fail. The proposed method utilises soft data from the iterative IDMA receiver to effectively cancel interference from the co-channel users. Analytical performance in terms of the number of users, spreading gain, performance of the IDMA receiver, and noise variance is developed, together with simulation results.
\end{abstract}

\section{INTRODUCTION}

Efficient timing acquisition in Interleaved Division Multiple Access (IDMA) systems is essential for the conventional IDMA detection scheme which is synchronous [6]. Minimising false alarms and missed detections are also important functions of the acquisition unit. The acquisition function is to determine the interleaving/chip timing to within a half a chip interval, such that the interleavers are synchronous with the user data. It has been shown that in fully loaded systems with high levels of multiple access interference, poor acquisition performance can severely reduce the capacity of multiple user systems [1]. Timing acquisition techniques for DS-CDMA systems have been widely studied. In [3] [4] the authors define the capacity of multiple access CDMA systems while maintaining acceptable performance. However, these studies often assume low levels of multiple-access interference (MAI). In [1] [2] the authors develop a model for acquisition using soft interference cancellation techniques for DS-CDMA. IDMA can be seen as a variant of DS-CDMA where the order of interleaving and spreading functions are swapped. Therefore we are motivated to apply acquisition techniques similar to those detailed in [1] [2] for IDMA acquisition.

In this paper we develop acquisition techniques for IDMA that work under high interference scenarios. The type of situations we are interested in is where the number of users equals the spreading gain. Since IDMA has been shown to outperform CDMA under high MAI scenarios [6], we expect acquisition techniques based on IDMA interference cancellation to outperform those for CDMA [2], in terms of probability of false alarms and missed detections for the same $E_{b} / N_{0}$ and system load.
We describe here a data directed acquisition system where the intermediate decoding information is used to assist detecting new users. This method is commonly known as soft interference cancellation. The method is independent of the specific acquisition technique[2], however for analysis and simulation in this paper we use the simple correlator techniques [7].

The paper is organized as follows. In Section II we discuss the system model. Section III describes the data directed acquisition approach. An analytical study is detailed in Section IV before analytical and simulation results are presented in Section V. Finally conclusions are drawn in Section VII.

\section{SYSTEM MODEL}

We employ an IDMA system [5] [6] where $K+1$ users each transmit symbols $d_{k}(n)$. For the purpose of acquisition it is important that each user have a unique random spreading code $\left(s_{k}(l)\right)$. In the model user $k$ is allocated the random interleaver $\pi_{k}$. The model assumes that no modulation is used for the new user as in the preamble for 3GPP [9] $\left(d_{K+1}(1)=1\right)$.

The transmitted baseband signal at chip interval $j$ according to the IDMA model is then

$$
y(j)=\sum_{k=1}^{K} h_{k} x_{k}(j)+x_{K+1}(j)
$$

Where $x_{k}(j)=\pi_{k}\left(s_{k}(l) d_{k}(n)\right)$,

$x_{K+1}(j)=\pi_{K+1}\left(s_{K+1}(l)\right), n=\left\lceil\frac{l}{N}\right\rceil$ and $h_{k}=1$ represents the channel coefficient for user $k$. Note that the index $l$ is necessary since chip indexes are altered as result of interleaving.

The channel adds zero mean complex white Gaussian noise (AWGN) with variance $\sigma^{2}=N_{0} / 2$, where $N_{0}$ is the single sided noise power spectral density. The channel output is therefore,

$$
\begin{aligned}
r(j) & =\sum_{k=1}^{K} \pi_{k}\left(s_{k}(l) d_{k}(n)\right)+\pi_{K+1}\left(s_{K+1}(l)\right)+n(j) \\
& =\xi_{K+1}(j)+\pi_{K+1}\left(s_{K+1}(l)\right)+n(j)
\end{aligned}
$$

where the noise term $n(j)$ is normally distributed $N\left(0, \sigma^{2}\right)$. The first term in (2) describes $K$ interfering users where the MAI is $\xi_{k}(j)$ and the second term is the signal of the new user. Without loss of generality synchronous chip timing is assumed, therefore square chip pulses are used. As random spreading codes are used the same result would be expected 
for a symbol asynchronous system. We assume that there is perfect carrier synchronization and that there is no frequency uncertainty. The results, however, are easily extendable to systems that assume frequency offsets. The system model also assumes no multipath, however, assuming the system is power constrained and because of the use of random codes, the interference levels would be similar [2], therefore little difference would be expected in the performance results. The system assumes no time variation during acquisition, such as time drift or change in channel conditions, which would be tracked within a timing synchronisation unit.

\section{ACQUisition with Iterative MUD Under High MAI}

In conditions where the number of users $(K)$ equals the spreading gain $(N)$ traditional code acquisition techniques based on correlating techniques fail [4]. This is because the MAI is too severe, resulting in low cross-correlation between the correlator and the desired signal. As a direct result acquisition performance suffers from noise and interference from other users. In the uplink of a mobile cellular system the base station receiver is assumed to use an IDMA multiuser receiver [6]. The conventional IDMA receiver computes soft information of all users being detected. These signals cause the MAI in the acquisition module, which is looking for users not yet known to the receiver.

Initially the base station is turned on and no terminals are connected, over time users connect and disconnect. The receiver estimates the transmitted signals from all connected users, using the known channel state information. If the receiver cancels estimated data of all connected users from the received baseband signal, what is left is system noise, residual interference, and signals from unknown users. The received data from connected users must meet frame error rate requirements, therefore their data estimates are accurate enough to be used for interference cancelation. If this was not the case then the user would be dropped as part of the Radio Link Control functionality [2].

We assume the received spread signal, $r(j)$, now consists of both signals that are being tracked by the receiver $\hat{\xi}_{K+1}(j)=\mathrm{E}\left[\xi_{K+1}(j)\right]$, and the desired user $x_{K+1}(j)$. The decision directed approach then computes a residual term,

$$
\eta_{K+1}(j)=r(j)-\hat{\xi}_{K+1}(j)
$$

Note that the chip values of $\eta_{K+1}(j)$ are taken over one symbol length, $N$, within a single IDMA block. A term which is of equivalent value (used for mathematical analysis) can be written,

$$
\eta_{K+1}^{\prime}(l)=\pi_{K+1}^{-1}\left(r(j)-\hat{\xi}_{K+1}(j)\right)
$$

Where $\xi_{K+1}(j)$ is the sum of the estimate of the spread signal sent by users 1 to $K$. The added complexity of computing $\eta_{K+1}(j)$ is very low due to the use of an IDMA IMUD. Figure 1 shows the implementation of the IDMA acquisition unit. Essentially the idea is that soft information from the
IMUD, which is the estimate of the MAI is subtracted from the received signal. If the receivers estimation is perfect the remaining signal only contains noise and signal from new users. A correlator can then be used to acquire these signals. It is important to note that in our system model the correlator is performed for a single symbol period $\left(d_{K+1}(n)\right)$. Since the IDMA block is composed of chips for $L$ QPSK symbols, the interleaver length is $L N$. This means that the desired signal, is interleaved over chip interval from 1 to $L N$. Therefore a correlation mask must be constructed,

$$
\begin{aligned}
s_{K+1}^{\prime}(l) & = \begin{cases}s_{K+1}(l-(n-1) N), l=(n-1) N+1 \ldots n N \\
0, & \text { otherwise }\end{cases} \\
x_{K+1}^{\prime}(j) & =\pi_{K+1}\left(s_{K+1}^{\prime}(l)\right)
\end{aligned}
$$

It is clear that the performance of the system relies heavily on the performance of the IMUD.

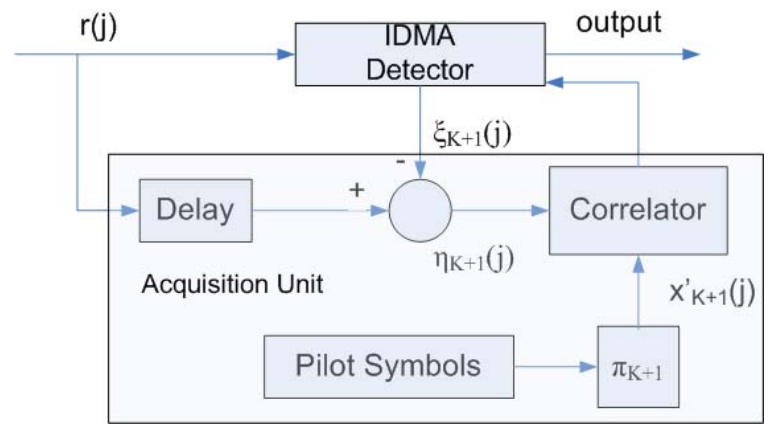

Fig. 1. IDMA Receiver with Acquisition unit

Figure 1 also highlights the need for a delay between input signal and the cancellation process. This is because the IMUD requires a certain amount of time to determine its estimate of the received signal.

In the correlator, the residual term given in (3) is correlated with the desired users signal, such that the timing position can be determined. This method enhances the conventional correlation approach by use of soft information. The resultant signal can be written (Assuming that we are trying to determine the first symbol in the IDMA block, $d_{K+1}(n)$ ).

$$
r_{p}=\left|\sum_{j=1}^{L N} \eta_{K+1}(j) x_{K+1}^{\prime}(j)\right|
$$

For mathematical analysis (6) can be simplified using (5),

$$
r_{p}=\left|\sum_{j=1}^{N} \eta_{K+1}^{\prime}(j) s_{K+1}(j)\right|
$$

\section{ANALYSIS}

In this section the goal is to analyse the performance of the proposed system in terms of false alarm and missed detection probabilities. The false alarm probability $\left(P_{f a}\right)$ is defined as the probability of the signal level being above 
the threshold where the timing point is incorrect. The missed detection probability $\left(P_{m d}\right)$ is defined as the probability of the signal level being below the threshold when the timing point is correct. This result is parameterised on the number of users, processing gain, noise variance and the cancellation factor of the system. In [6] the IDMA detector performs soft computation of the first and second order statistics of the interference. This technique can be applied in the realization of the above mentioned acquisition unit. In this section we take this result one step further and analytically determine the probability density function of the cross correlation term (7).

To start with we need to determine the first and second order statistics of the residual term (4). It is sufficient to consider only the distribution of the real metric,

$$
\begin{aligned}
\eta_{K+1}^{R e}(j) & =\sum_{k=1}^{K}\left(x_{k}^{R e}(j)-\tilde{x}_{k}^{R e}(j)\right)+x_{K+1}^{R e}+n(j) \\
\mathrm{E}\left[\eta_{K+1}^{R e}(j)\right] & =s_{K+1}(l) \\
\operatorname{Var}\left(\eta_{K+1}^{R e}(j)\right) & =\sum_{k=1}^{K}\left\|s_{k}(l)\right\|^{2} \mathrm{E}\left[\left(d_{k}^{R e}(i)-\tilde{d}_{k}^{R e}(i)\right)^{2}\right]+\sigma_{n}^{2} \\
& =\frac{K}{N} \sigma_{d}^{2}+\sigma_{n}^{2}
\end{aligned}
$$

Where $s_{k}(j)$ are i.i.d and take values $\pm \frac{1}{\sqrt{N}}, l$ is the inverse permutation of the index set $j$ (i.e. $s_{K+1}(l)=$ $\pi_{K+1}^{-1}\left(x_{K+1}(j)\right), i$ is an element of the data block (i.e. $i \in\{1 \ldots L\}), \sigma_{d}^{2}=\mathrm{E}\left[\left(d_{k}^{R e}(i)-\tilde{d}_{k}^{R e}(i)\right)^{2}\right]$ and $\tilde{d}_{k}(n)=$ $E\left[d_{k}^{\operatorname{Re}}(n)\right]+j E\left[d_{k}^{\operatorname{im}}(n)\right]$.

Thus, the distribution of the residual term given in (4) can be approximated by a complex gaussian distribution with mean, $\mu_{\eta}=s_{K+1}(l)+j s_{K+1}(l)$ and variance, $\sigma_{\eta}=\frac{K}{N} \sigma_{d}^{2}+\sigma_{n}^{2}$ in each dimension.

\section{A. Analysis of Off-time Density}

We now develop a method to determine the density of the off-time signal, that is, where the correlator sequence is not time matched with the transmitted sequence. As the codes are random this is equivalent to determining the density of any random code convolved with (4), in the following analysis we use a hypothetical user (user $K+2$ ). We know that the distribution of $\eta_{K+1}^{R e}(j)$ which is a complex gaussian distributed random variable. Now consider the cross correlation term (7) during off-time,

$$
\mathrm{r}_{p, \text { off }}=\sum_{l=1}^{N} \eta_{K+1}^{\prime}(l) s_{K+2}(l)
$$

Where $\eta_{K+1}^{\prime}(j)=\sum_{k=1}^{K} s_{k}\left(d_{k}(i)-\tilde{d}_{k}(i)\right)$. According to the central limit theorem, $r_{p \text {,off }}$ can be seen as a complex gaussian random variable. We first determine the distribution of the term $r_{p \text {,off }}^{\mathrm{Re}}$ by computing its mean and variance.

$$
\begin{aligned}
\mathrm{E}\left[r_{p, \text { off }}\right] & =\mathrm{E}\left[\sum_{l=1}^{N} \eta_{K+1}^{\prime}(l) s_{K+2}(l)\right] \\
& =0 \\
\operatorname{Var}\left(r_{p, \text { off }}\right) & =\sum_{l=1}^{N} \operatorname{Var}\left(s_{K+2}(l) \eta_{K+1}^{\prime}(l)\right) \\
& =\frac{K \sigma_{d}^{2}+1}{N}+\sigma_{n}^{2}
\end{aligned}
$$

Thus, the real valued distribution is given by,

$$
f_{r_{p, \text { off }}^{\mathrm{Re}}}(r)=\frac{1}{\sqrt{2 \pi \sigma_{r_{p}}^{2}}} \exp \left(-\frac{r^{2}}{2 \sigma_{r_{p}}^{2}}\right)
$$

Where $\sigma_{r_{p, \text { off }}}^{2}=\operatorname{Var}\left(r_{p, \text { off }}\right)$, and the imaginary distribution $f_{r_{p, \text { off }}}\left(r^{\mathrm{Im}}\right)$ is given by a similar density function.

Utilising a result in [8] the term $\left|r_{p}\right|$ is given by a Rayleigh distribution, with parameter, $\sigma_{\text {off }}^{2}=\sigma_{\mathrm{r}_{\mathrm{p}}}^{2}$,

$$
f_{\left|r_{p, \text { off }}\right|}(r)=\frac{r}{\sigma_{\text {off }}^{2}} \exp \left(-\frac{r^{2}}{2 \sigma_{\text {off }}^{2}}\right)
$$

We have now developed the so-called "off-time" density as a function of the number of users, $K$, the spreading factor $N$, the noise variance $\sigma_{n}^{2}$, the variance of the soft output estimator $\sigma_{d}^{2}$ and a threshold value of $r_{p}$. This density will be later used to determine the false alarm probability $\left(P_{f a}\right)$.

\section{B. Analysis of On-time Density}

We now derive the on-time density. The real valued Gaussian distribution of the peak correlation function during ontime is given by,

$$
r_{p, \mathrm{on}}^{R e}=\sum_{l=1}^{N} \eta_{K+1}^{\prime}(l) s_{K+1}(l)
$$

And the first and second order statistics of $r_{p \text {,on }}^{R e}$ are computed,

$$
\begin{aligned}
\mathrm{E}\left[r_{p, \text { on }}^{\mathrm{Re}}\right] & =\sum_{l=1}^{N} \mathrm{E}\left[s_{K+1}(l) \eta_{K+1}^{\prime}(l)\right] \\
& =1 \\
\operatorname{Var}\left(r_{p, \text { on }}^{\mathrm{Re}}\right) & =\sum_{l=1}^{N} \operatorname{Var}\left(s_{K+1}(l) \eta_{K+1}^{\prime}(l)\right) \\
& =\frac{K \sigma_{d}^{2}}{N}+\sigma_{n}^{2}
\end{aligned}
$$

Therefore, the term $r_{p \text {,on }}^{R e}$ has real-time density function given by,

$$
f_{r_{p, \mathrm{on}}^{R \mathrm{e}}}(r)=\frac{1}{\sqrt{2 \pi \sigma_{r_{p, \mathrm{on}}^{\mathrm{Re}}}^{2}}} \exp \left(-\frac{(r-1)^{2}}{2 \sigma_{r_{p, \mathrm{on}}^{\mathrm{Re}}}^{2}}\right)
$$

Where $\sigma_{r_{p \text { on }}^{\mathrm{Re}}}^{2}=\operatorname{Var}\left(r_{p, \text { on }}^{\mathrm{Re}}\right)$.

This density represents the random variable in the real axis, a similar density function can be formulated for the imaginary 
axis. To convert this to a density which represents the absolute value of the signal we utilise a result the Rician distribution [8]. Therefore the "on-time" distribution is,

$$
f_{\left|r_{p, \text { on }}\right|}(r)=\frac{r}{\sigma_{\text {on }}^{2}} \exp \left(-\frac{r^{2}+s^{2}}{2 \sigma_{\mathrm{on}}^{2}}\right) \mathrm{I}_{0}\left(\frac{r s}{\sigma_{\mathrm{on}}^{2}}\right)
$$

Where $\sigma_{\text {on }}=\frac{K \sigma_{d}^{2}}{N}+\sigma_{n}^{2}, s^{2}=2$ and $\mathrm{I}_{0}(\cdot)$ is the zero order modified Bessel function of the first kind.

\section{False alarm / Missed Detection probabilities}

Now we have derived the probability densities we wish to integrate these functions to determine the probabilities of the missed detection and false alarms. This means we need to integrate (16) from $0 \rightarrow$ a to determine the probability of missed detection, where a is the detection threshold used to acquire a new user. Similarly, we need to integrate (12) from $\mathrm{a} \rightarrow \infty$ to the determine probability of false alarm. Starting first with (16)

$$
\begin{aligned}
\mathrm{P}_{m d} & =\operatorname{Pr}(0<r<a) \\
& =\int_{0}^{a} \frac{r}{\sigma_{\text {on }}^{2}} \exp \left(-\frac{r^{2}+s^{2}}{2 \sigma_{\text {on }}^{2}}\right) \mathrm{I}_{0}\left(\frac{r s}{\sigma_{\text {on }}^{2}}\right) d r
\end{aligned}
$$

The probability of missed detection is therefore the CDF of the rician density given (16) which is given in [8] as

$$
\mathrm{P}_{m d}=1-\mathrm{Q}_{1}\left(\frac{s}{\sigma_{\mathrm{on}}}, \frac{a}{\sigma_{\mathrm{on}}}\right), a \geq 0
$$

Where $\mathrm{Q}_{1}(\cdot)$ is the generalised Marcum's $\mathrm{Q}$ function where $m=\frac{n}{2}=1$ as the degrees of freedom is $n=2$. Therefore

We now derive the probability of false alarm by integrating the Rayleigh distribution from (12), therefore

$$
\begin{aligned}
\mathrm{P}_{f a} & =\operatorname{Pr}(R>a) \\
& =\exp \left(-\frac{a^{2}}{2 \sigma_{\text {off }}^{2}}\right)
\end{aligned}
$$

\section{Determining Residual Interference}

The residual interference from the IMUD receiver will determine the performance of the acquisition unit. The purpose of this section is to determine a typical residual interference level $\left(\sigma_{d}^{2}\right)$, in order to achieve the expected performance of the system. The typical frame error rate (FER) that maximizes capacity in a cellular environment has been arguably shown to be approximately $\mathrm{FER}=0.1$ [9]. We assume that the bit error rate is equal to or lower than $10^{-4}$, based on a block size of 1000 . We represent the soft values prior to a decision as gaussian distributed random variables, where $\left.\operatorname{Pr}\left(d_{k}(n)<0 \mid d_{k}(n)=+1\right)=\mathrm{P}_{e}=\mathrm{BER}\right)$. We now wish to determine $\sigma_{d}^{2}$ in terms of $\mathrm{P}_{e}$ using the assumption above and that IDMA achieves single user performance. We know from [8] that

$$
\sigma_{d}=\frac{\sqrt{\varepsilon_{b}}}{\mathrm{Q}^{-1}\left(\mathrm{P}_{e}\right)}
$$

Therefore for a probability of error, $\mathrm{P}_{e}=3.87 \times 10^{-6}$ the variance estimate is $\sigma_{d}^{2}=0.05$ for an $E_{b} / N_{0}=10 \mathrm{~dB}$, and for a $\mathrm{P}_{e}=7.27 \times 10^{-4}$ the variance estimate is $\sigma_{d}^{2}=0.1$ corresponding to an $E_{b} / N_{0}=7 \mathrm{~dB}$. From simulation we validated the theoretical computation of $\sigma_{d}$ in (20) as compared with the cancellation factor $\left(\sigma_{d}\right)$ during simulation. This is expected under good channel conditions where the IDMA IMUD achieves near single user performance. We will utilise these figures to determine expected performance of the acquisition approach defined.

\section{Analytical and Numerical performance RESULTS}

In this section we show results from both our analytical development and from our simulation. The simulation consists of transmitting 2000 blocks where the codes/interleavers for the multiple access interference are randomly selected for each user. In all cases the spreading gain was chosen to be $N=16$ and the IDMA receiver was run for 10 iterations. However it has been shown that IDMA has equivalent or better performance as $N$ increases due to the increased interleaver lengths (resulting in better turbo decoding performance). The system is chip and symbol synchronous and all users have the same power. However, the acquisition unit could conceivably used in conjunction with a timing synchronisation unit.

Figure 2 shows the on and off-time distributions for three different scenarios at an $\mathrm{E}_{\mathrm{b}} / \mathrm{N}_{0}=7 \mathrm{~dB}$. The first plot is the single user(SU) scenario, here the on-time density is centered around $\sqrt{2}$ and is Rician distributed while the off-time distribution is centered around $\frac{1}{2 \sqrt{2}}$ and is Rayleigh distributed. For the single user case there is a distinct separation between the two densities, for example, a threshold set around $\mathrm{a}=0.85$ would provide both a low false alarm probability and a low missed detection probability. In practical systems the threshold is determined for a particular $\mathrm{P}_{f a}$, this sets the amount of time the receiver will be occupied processing false alarms. The choice of $a$ determine the trade-off between false alarms and missed detections.

In the last subplot the densities for the full interference(FI) case with $\mathrm{K}=16$ users in the system is shown. The full interference case is where no soft information is used for interference cancellation $\left(\tilde{d}_{K+1}(n)=0\right)$. As such the acquisition unit suffers from much more severe MAI. It is clear that a logical threshold point is not achievable as the on and off-time densities are not separated, this will also be seen later in Figure 3. In the second subplot the densities for $\mathrm{K}=16$ are shown, however partial cancellation (PC) is used where $\sigma_{d}^{2}=0.1$. It can be seen that the resultant densities are significantly improved such that they are equally as distinct as the single user case due to the near single user performance of the IDMA IMUD [6]. This is where an IDMA IMUD outperforms CDMA, resulting in better IC (better cancellation 

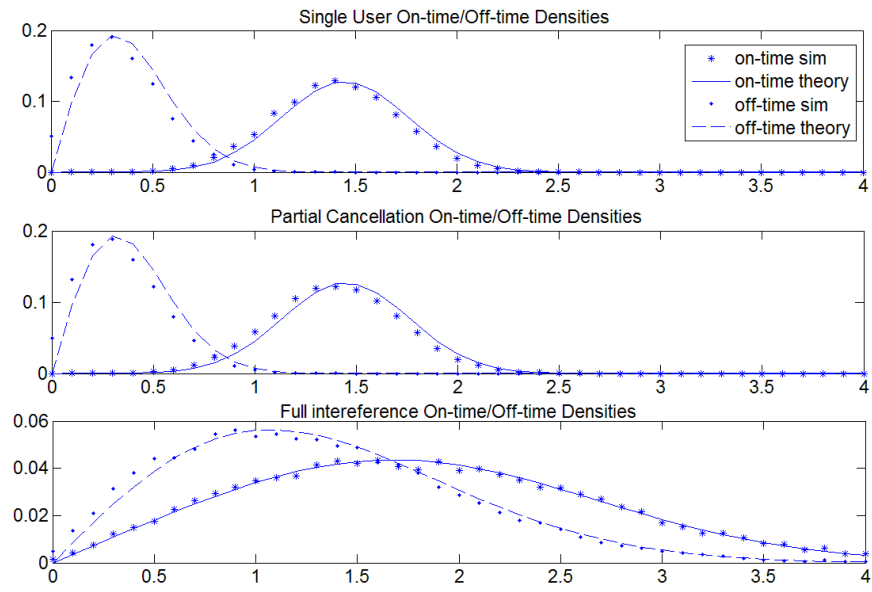

Fig. 2. On-off densities for different scenarios

factor $\sigma_{d}^{2}$ ) for heavily loaded multiple user systems. For the same reasons it is expected that it is possible to perform acquisition (with near single user performance) for overloaded systems using the IDMA IMUD.

In Figure 3 we show the performance of the full interference and two partial cancellation cases $\left(\sigma_{d}^{2}=0.1\right.$ and $\left.\sigma_{d}^{2}=0.05\right)$. We compare the analytical result (solid line) to the simulation results (points). The plot is for an integration period of 1 symbol, with $\mathrm{E}_{b} / \mathrm{N}_{0}=7 \mathrm{~dB}$ for $\sigma_{d}^{2}=0.1$ and $\mathrm{E}_{b} / \mathrm{N}_{0}=10 \mathrm{~dB}$ for $\sigma_{d}^{2}=0.05$. As can be seen the performance of the partial cancellation scheme is substantially better than that of the fully loaded system without cancellation. For example fixing $\mathrm{P}_{f a}=10^{-4}$ the partial cancellation results are $\mathrm{P}_{m d}=0.03$ and $\mathrm{P}_{m d}=0.5$ for $\sigma_{d}^{2}=0.05$ and $\sigma_{d}^{2}=0.1$ respectively. In comparison to the fully loaded system where $\mathrm{P}_{m d}=1$, i.e. $100 \%$ of detections would be missed.

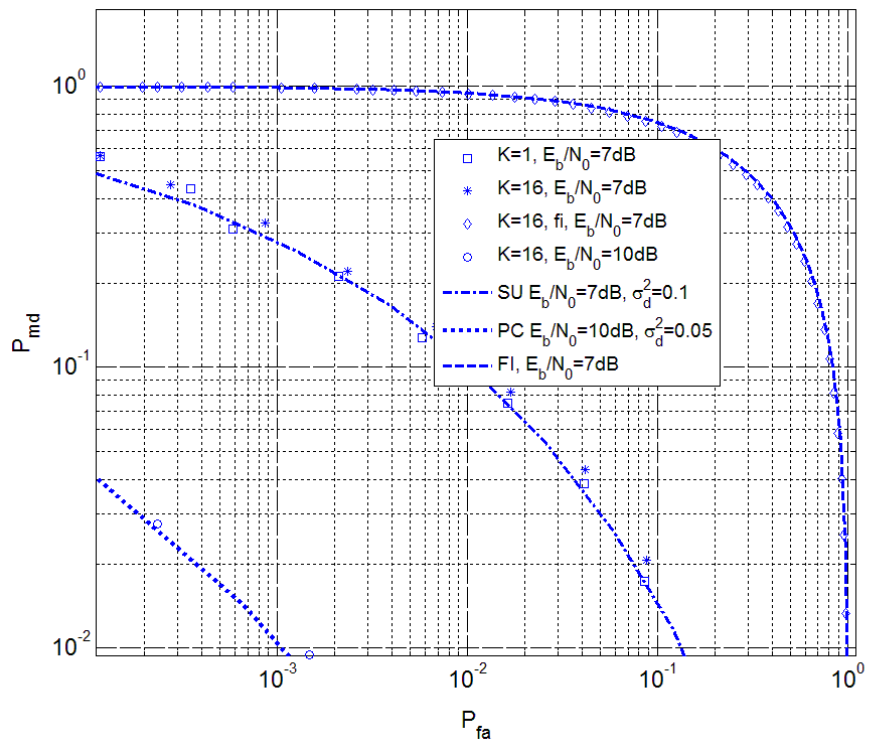

\section{CONCLUSION}

In this paper we have investigated an acquisition technique based on soft interference cancellation applied to IDMA. This has been performed under severe multiple access interference.

We derived density functions to represent the so-called ontime and off-time random variables and we compare these densities to simulation results. These densities are functions of the number of users, the processing gain, the cancellation factor and the noise variance. We integrated these densities to determine the probability of false alarm and probability of missed detection, as a function of threshold value. We compared both analytical results and simulation results for $P_{f a}$ vs. $P_{m d}$ for $K=16$ users with partial cancellation from the IDMA receiver. The results show that acquisition using conventional correlation techniques for CDMA is not practical when the number of users equals the spreading gain, however, when utilising the IDMA IMUD good performance of a conventional correlator for acquisition is possible.

In conclusion this paper demonstrated that for high performance multiuser detection, information sharing between the correlator unit and the receiver is essential to perform acquisition of new users. Depending on the reliability of the information from the IMUD, this technique improves the system capacity by reducing the amount of time spent processing false alarms and the number of missed detections.

\section{ACKNOWLEDGMENT}

Bathiya Senanayake is with the Australian National University, Mark C. Reed and Zhenning Shi are with the National ICT Australia and affiliated with the Australian National University. National ICT Australia is funded through the Australian Governments Backing Australias Ability initiative and in part through the Australian Research Council.

\section{REFERENCES}

[1] M. C. Reed and L. W. Hanlen, "Return link code acquisition for DSCDMA in a high capacity multi-user systems," In Proc. IEEE Int. Symp. on Spread Spectrum Techniques and Apps, pp. 218-222, August 2004.

[2] M. C. Reed and L. W. Hanlen, "Return link code acquisition for 1-D and 2-D with DS-CDMA for High Capacity Multiuser Systems," IEEE Trans. on Vehicular Technology, pp. -, March 2006.

[3] U. Madhow and M. B. Pursley, "Acquisition in direct sequence spread spectrum communication networks: An asymptotic analysis," IEEE Trans. Inform. Theory, vol. 39, no. 903-912, May 1993.

[4] G. Corazza and V. Degli-Espoti, "Acquisition based capacity estimates for cdma with imperfect power control," in IEEE int. Symp. on Spread Spectrum Techniques and Applications, pp. 325-329, 1994.

[5] W. K. Leung, L. Liu, and L. Ping, "Interleaving-based multiple access and iterative chip-by-chip multiuser detection," IEEE Commun. Magazine, pp. S19-S23, 2005.

[6] L. Ping, L. Liu, K. Y. Wu, and W. K. Leung, "Interleave division multiple access (IDMA) communications," Proc. 3rd International Symposium on Turbo Codes and Related Topics, pp. 173-180, 2003.

[7] R. L. Pickholtz, D. L. Schilling, and L. Milstein, "Theory of spreadspectrum communications-a tutorial," IEEE Trans. Commun, vol. 30, pp. 855-884, May 1982.

[8] J. G. Proakis, Digital Communications, 3rd ed. McGraw-Hill, 1995.

[9] H. Holma and A. Toskala, WCDMA for UMTS. West Sussex, England:Wiley, 2002.

Fig. 3. Performance of the Acquisition unit 